\title{
Deployment analysis and optimization of heterogeneous networks under the spectrum underlay strategy
}

\author{
Anabel Martínez-Vargas ${ }^{*}$ and Ángel G Andrade
}

\begin{abstract}
To meet the growing mobile traffic demand, deployment of heterogeneous networks (HetNets) is proposed as a solution to enhance network capacity. It will enable the coexistence of several systems consisting of Macro, Micro, and Femto or WiFi layers. However, the main issue is the coexistence due to mutual interference. To guarantee that HetNet performance will not be compromised due to the interference, some deployment parameters should be controlled, such as primary exclusion zones and density of users. In this context, the present work provides deployment analysis and optimization for a Macro-Femto scenario, considering interference constraints to maximize the data rate of the HetNet. To fulfill this, we characterize some spectrum underlay scenarios in which HetNets would be expected to operate, setting different signal-to-interference-plus-noise ratio (SINR) thresholds. They are evaluated using a solution procedure based on the socio-cognitive particle swarm optimization (SCPSO) algorithm. Considering the best scenarios found, we show deployment parameters to achieve maximum system throughput, such as density of femto-users and primary exclusion zones of macro-users.
\end{abstract}

Keywords: Deployment parameters; Heterogeneous network; Spectrum underlay

\section{Introduction}

Architecture of traditional cellular networks has been undergoing dramatic changes due to the intense consumer demand for mobile data. Recently, the heterogeneous networks (HetNets) have emerged as the network architecture to support the growing demand for data services. They have a hierarchical architecture, comprising an overlay of a macrocell network with additional tiers of small cells (picos, femtos, or WiFi access points). This enlarges the pool of available spectrum resources for mobile users, since they are reused across the multiple tiers in the HetNet, harnessing also an additional spectrum in unlicensed bands by integrating WiFi hotspots (femtocells) in the network. The use of WiFi in conjunction with the Macrocell allows relieving congestion on it. From the above and the cost point of view, WiFi has become an integral part of the operator's strategy [1]. However, by reusing resources, potentially destructive interference in the HetNet is generated.

\footnotetext{
* Correspondence: martinez.anabel@uabc.edu.mx

College of Engineering, MyDCl, Autonomous University of Baja California (UABC), Blvd. Benito Juárez, Mexicali 21280, México
}

Unlike macro base stations (macro-BSs), which are installed according to detail network planning, operators have limited control over femto base stations (femto-BSs) since they are deployed by the end-users. This leads to two kinds of interference between macro and femto cells: cross-tier (the aggressor and the victim of interference belong to different tiers) and intra-tier (the aggressor and the victim of interference belong to the same tier) [2]. Cognitive radio (CR) technology can address those issues, providing to femto-BS capabilities to sense the environment, interpret the received signal from the macro-BS and the surrounding femto-BSs, and intelligently allocate spectrum resources while cross-tier and the intra-tier interference are controlled. The set of techniques to share and allocate spectrum resources through CR technology is known as dynamic spectrum access, where the users with low priority are known as secondary users (femtocells), whereas the members of the prioritized user group are named primary users (macrocell) [3]. To access the cellular channels (or primary channels), a secondary user performs one of the following dynamic spectrum access techniques:

\section{穴}


transmit simultaneously with the primary user as long as the resulting interference is constrained (spectrum underlay), or exploit an unused channel of the primary user (spectrum overlay) [2]. Therefore, the underlay scheme has a more efficient reuse of spectral resources since one primary channel is exploited simultaneously by two or more users. Furthermore, the spectrum sensing operation of the overlay scheme requires complicated computation at the secondary transmitter.

Identifying deployment parameters for HetNets help to prevent the generation of harmful interference between heterogeneous wireless systems that interact with each other. Interference is considered harmful if it causes disruption in user's service. In general, examples of deployment parameters for HetNets are $[4,5]$ primary exclusion zones (radius of protection of a primary receiver/transmitter), transmission power limits on secondary users, number of admitted secondary users, acceptable level of interference in primary receptor, and monitoring time. The deployment parameters depend on the dynamic spectrum access technique (overlay or underlay) that a secondary user performs to exploit a primary band. In the case of deploying a HetNet with spectrum underlay access, it is relevant to set a limit on the transmission power of secondary users, primary exclusion zones, permissible number of secondary users, and acceptable level of interference in the primary receptor. The detection threshold and monitoring time are factors to overlay access mode. The first refers to the maximum value to be set to detect the primary signal; therefore, the primary channel status is known (busy or idle). In contrast, the second refers to the time that a secondary user should expend to detect the presence of the primary user.

In this paper, we characterize some spectrum underlay scenarios in which HetNets would be expected to operate under different signal-to-interference-plus-noise ratio (SINR) thresholds. They are evaluated using our proposed access control and channel assignment algorithm presented in [6], which is a solution procedure with less complexity based on an improved version of binary particle swarm optimization (BPSO) algorithm called socio-cognitive particle swarm optimization (SCPSO) [7]. Considering the best scenarios, the deployment parameters to achieve maximum system throughput, such as the number of admitted secondary users coexisting with primary users and primary exclusion zones, are presented. Particularly, we focus on the Macro-Femto scenario in which the secondary network (femtocell) operates in an unlicensed band; while the primary network (macrocell) is composed by devices transmitting on Long Term Evolution Release 8 and beyond (LTE-Release 8).

Some works have addressed the specifications of network deployment using mainly statistical models such as in [8] in which from a log-normal distribution, the authors find radius of exclusion zones and density of secondary users. In this context, in [9], an outage-based distributed user removal algorithm to devise the number of secondary users in a spectrum underlay HetNet is presented. In [10], a tool for estimating the protection distance around the primary transmitter by means of gamma and normal distributions is proposed. Although the

Table 1 Symbols used in the access control and channel assignment algorithm

\begin{tabular}{|c|c|}
\hline Symbols & Meaning \\
\hline $\mathrm{SINR}_{u}$ & The SINR at each secondary link $u$ \\
\hline$P_{u}$ & The transmit power of secondary transmitter $u$ \\
\hline$P_{k}$ & The transmit power of secondary transmitter $k$ \\
\hline$P_{v}$ & The transmit power of primary transmitter $v$ \\
\hline Ids $(u)$ & The link distance of secondary link $u$ \\
\hline$d s s(k, u)$ & $\begin{array}{l}\text { The distance from secondary transmitter } k \text { to } \\
\text { secondary receiver } u\end{array}$ \\
\hline$d p s(v, u)$ & $\begin{array}{l}\text { The distance from primary transmitter } v \text { to } \\
\text { secondary receiver } u\end{array}$ \\
\hline k & The index of active secondary transmitters \\
\hline$\Phi$ & The set of active secondary transmitters \\
\hline Y & The path loss exponent (a value between 2 and 4) \\
\hline SI & The number of secondary links \\
\hline $\mathrm{SINR}_{V}$ & The SINR at each primary link $v$ \\
\hline$I d p(v)$ & The link distance of primary link $v$ \\
\hline$d p s(k, v)$ & $\begin{array}{l}\text { The distance from secondary transmitter } k \text { to } \\
\text { primary receiver } v\end{array}$ \\
\hline$P l$ & The number of primary links \\
\hline$c_{u}^{\prime}$ & The data rate of the secondary link \\
\hline$c_{V}^{\prime \prime}$ & The data rate of the primary link \\
\hline$B$ & The primary channel bandwidth \\
\hline$a$ & The SINR threshold for the secondary network \\
\hline$\beta$ & The SINR threshold for the primary network \\
\hline$S$ & The swarm \\
\hline$X_{i}$ & The position of the ith particle \\
\hline$V_{i}$ & The velocity of the ith particle \\
\hline$V_{\max }$ & The maximum velocity \\
\hline$P_{i}$ & The best position ever visited by the ith particle \\
\hline$X_{i}^{\prime}$ & $\begin{array}{l}\text { The candidate channel allocation vector of the } \\
\text { ith particle }\end{array}$ \\
\hline$P_{i}^{\prime}$ & $\begin{array}{l}\text { The best channel allocation for secondary links } \\
\text { find so far by the ith particle }\end{array}$ \\
\hline$g$ & The index of the best particle in the swarm \\
\hline$T_{\max }$ & The number of iterations \\
\hline$c_{1}, c_{2}, c_{3}$ & The cognitive, social and socio-cognitive factors \\
\hline$w, w^{l}$ & The inertia weights \\
\hline$P C$ & The number of primary channels to share \\
\hline Spectrum status & The channel allocation vector for primary links \\
\hline$P_{g}$ & The best particle in the swarm \\
\hline
\end{tabular}




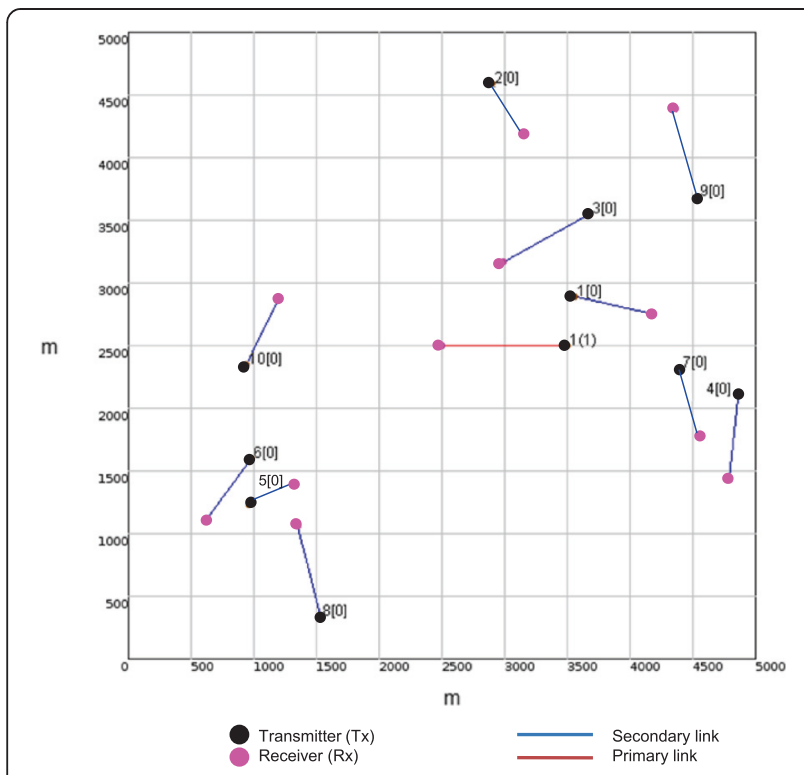

Figure $1 \mathrm{~A}$ snapshot sample before applying the access control and channel assignment algorithm based on SCPSO.

above works provide deployment parameters to guarantee primary network protection, our framework can suggest deployment parameters that ensure that the maximum throughput in the HetNet will be achieved, providing not only primary network protection but also secondary network protection. In others words, achieving the maximum throughput in the HetNet is possible as long as the deployment guidelines provided by our framework are applied. On the other hand, in [11], deployment issues on macro-pico heterogeneous networks are studied such as locations, density, and transmit power of pico eNodeBs (PeNBs) to offload traffic from the macro to pico cells to maximize overall throughput. To fulfill this, the cell range extension (CRE) is applied. Although it optimizes the aforementioned parameters, it first requires carefully setting the CRE bias value that implies also its optimization for a given scenario.

The novelty of our work is that it can suggest radius of exclusion zone and density of secondary users to achieve the maximum throughput in the HetNet with spectrum underlay, unlike the works cited above. The aim in works [8-11] is only to devise exclusion zones or permissible density of secondary users to offer protection to primary users. To the best of our knowledge, there is no effort to suggest exclusion zones and density of secondary users to achieve the maximum throughput in the context of an underlay spectrum sharing network. Instead, there are considerable efforts to let more wireless devices operate opportunistically within unused frequency bands referred as white spaces (overlay strategy).

The rest of this paper is organized as follows. The access control and channel algorithm is given in Section 2.

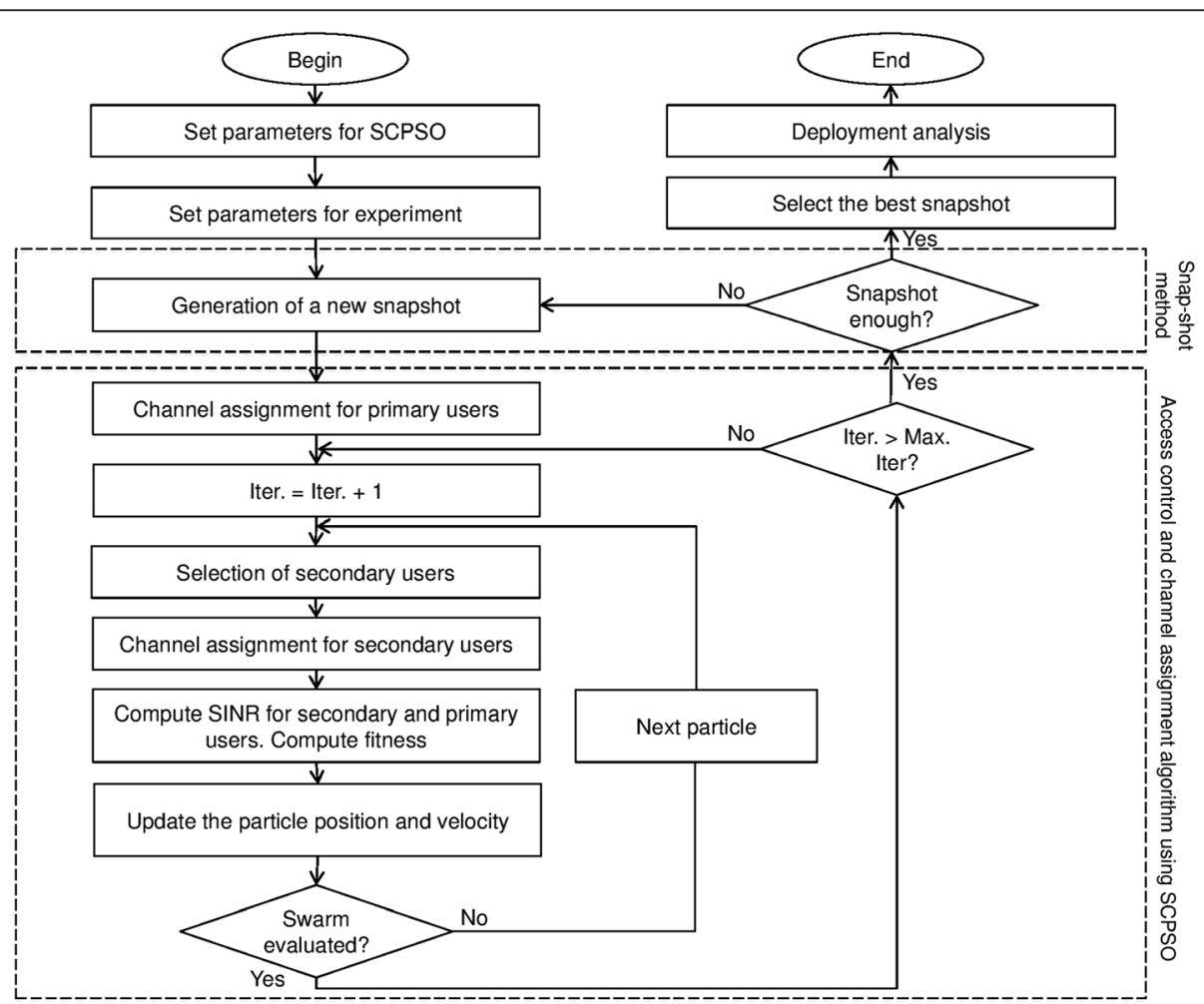

Figure 2 The process of simulation. 
Section 3 describes the simulation framework. Then, in Section 4, the deployment parameters of HetNets are presented. Finally, Section 5 gives the conclusion and future work.

\section{The access control and channel assignment algorithm}

To describe the development of the proposed algorithm, we used the symbols as defined in Table 1.

In the context of HetNets with spectrum underlay, when a secondary transmitter requests for a primary channel, they must be able to check if mutual interference among secondary users and primary users does not arise to the level of harmful interference. In other words, the HetNet must determine the new interference situation in the system if a primary channel is allocated to the requested secondary transmitter, to guarantee the quality of service (QoS) not only at the primary network but also at the secondary network. To fulfill this, the SINR is the most commonly studied model. In the SINR model, the energy of a signal fades with the distance to the power of the path loss parameter. If the signal strength received by a device divided by the interfering strength of other simultaneous transmissions (plus the fixed background noise) is above some reception threshold, then the receiver successfully receives the message; otherwise, it does not [12].

The SINR at each secondary link $u$ is given by:

$$
\operatorname{SINR}_{u}=\frac{P_{u} / l d s(u)^{\gamma}}{\sum_{k \in \Phi} P_{k} / d s s(k, u)^{\gamma}+P_{v} / d p s(v, u)^{\gamma}}, 1 \leq u \leq S l
$$

where $P_{u}$ is the transmit power of secondary transmitter $u$, $P_{k}$ is the transmit power of secondary transmitter $k, P_{v}$ is the transmit power of primary transmitter $v, l d s(u)$ is the link distance of secondary link $u, d s s(k, u)$ is the distance from secondary transmitter $k$ to secondary receiver $u, d p s$ $(v, u)$ is the distance from primary transmitter $v$ to secondary receiver $u, k$ is the index of active secondary transmitters, $\Phi$ is the set of active secondary transmitters, excluding the secondary transmitter $u, \gamma$ is the path loss exponent (a value between 2 and 4), and $S l$ is the number of secondary links deployed in the area.

On the other hand, to calculate the SINR at each primary link $v$, the following equation is considered:

$$
\mathrm{SINR}_{v}=\frac{P_{v} / l d p(v)^{\gamma}}{\sum_{k \in \Phi} P_{k} / d p s(k, v)^{\gamma}}, 1 \leq v \leq P l
$$

where $P_{v}$ is the transmit power of primary transmitter $v$, $P_{k}$ is the transmit power of secondary transmitter $k, l d p(v)$ is the link distance of primary link $v, d p s(k, v)$ is the distance from secondary transmitter $k$ to primary receiver $v$, and $P l$ is the number of primary links presented in the
Table 2 Parameters used for SCPSO

\begin{tabular}{ll}
\hline Parameters & Values \\
\hline Swarm size $S=$ & 40 \\
Number of iterations $T_{\max }=$ & 150 \\
Cognitive, social and socio-cognitive factors $C_{1}, c_{2}, c_{3}=$ & $2,2,12$ \\
Inertia weight $W=$ & 0.721 \\
Maximum velocity $V_{\max }=$ & {$[-6,6]$} \\
\hline
\end{tabular}

area. For purpose of our analysis, we only have one primary link; therefore, $P l=1$ and the index $v=1$.

Data rate contributions of the secondary links and primary links are calculated according to Equations 3 and 4, respectively. The data rate depends on primary channel bandwidth $B$ that secondary links and primary links can share and the conditions of the propagation environment (attenuation and interference).

$$
\begin{gathered}
c_{u}^{\prime}=B \log _{2}\left(1+\operatorname{SINR}_{u}\right) \\
c_{v}^{\prime \prime}=B \log _{2}\left(1+\operatorname{SINR}_{v}\right)
\end{gathered}
$$

Based on the above discussion, the access control and channel assignment in the HetNet is formulated as the following optimization problem:

$$
\operatorname{Max} \sum_{u=1}^{S l} c_{u}^{\prime} x_{u}+\sum_{v=1}^{P l} c_{v}^{\prime \prime}
$$

subject to:

$$
\begin{aligned}
& \operatorname{SINR}_{u} \geq \alpha \\
& \operatorname{SINR}_{v} \geq \beta \\
& c_{u}^{\prime}>0, u=1,2, \ldots, S l \\
& c_{v}^{\prime \prime}>0, v=1,2, \ldots, P l \\
& c_{u}^{\prime}, c_{v}^{\prime \prime} \in R^{+} \\
& x_{u}=\left\{\begin{array}{r}
1, \text { if } \operatorname{SINR}_{u} \geq \alpha \text { and } \operatorname{SINR}_{v} \geq \beta \\
0, \text { otherwise }
\end{array}\right.
\end{aligned}
$$

The objective function in (5) is to maximize the sum throughput in the HetNet. The SINR requirements of secondary links and primary links are posed in (6) and

Table 3 Parameters used for experiments

\begin{tabular}{ll}
\hline Parameters & Values \\
\hline Number of secondary links $S I=$ & $10,15,20,25$ \\
Number of primary links $P I=$ & 1 \\
Number of primary channels to share $P C=$ & 1 \\
Number of runs $=$ & 5,000 \\
SINR threshold $a, \beta(\mathrm{dB})=$ & $4,6,8,10,12,14$ \\
Primary channel bandwith $B=$ & $20 \mathrm{MHz}$ \\
\hline
\end{tabular}




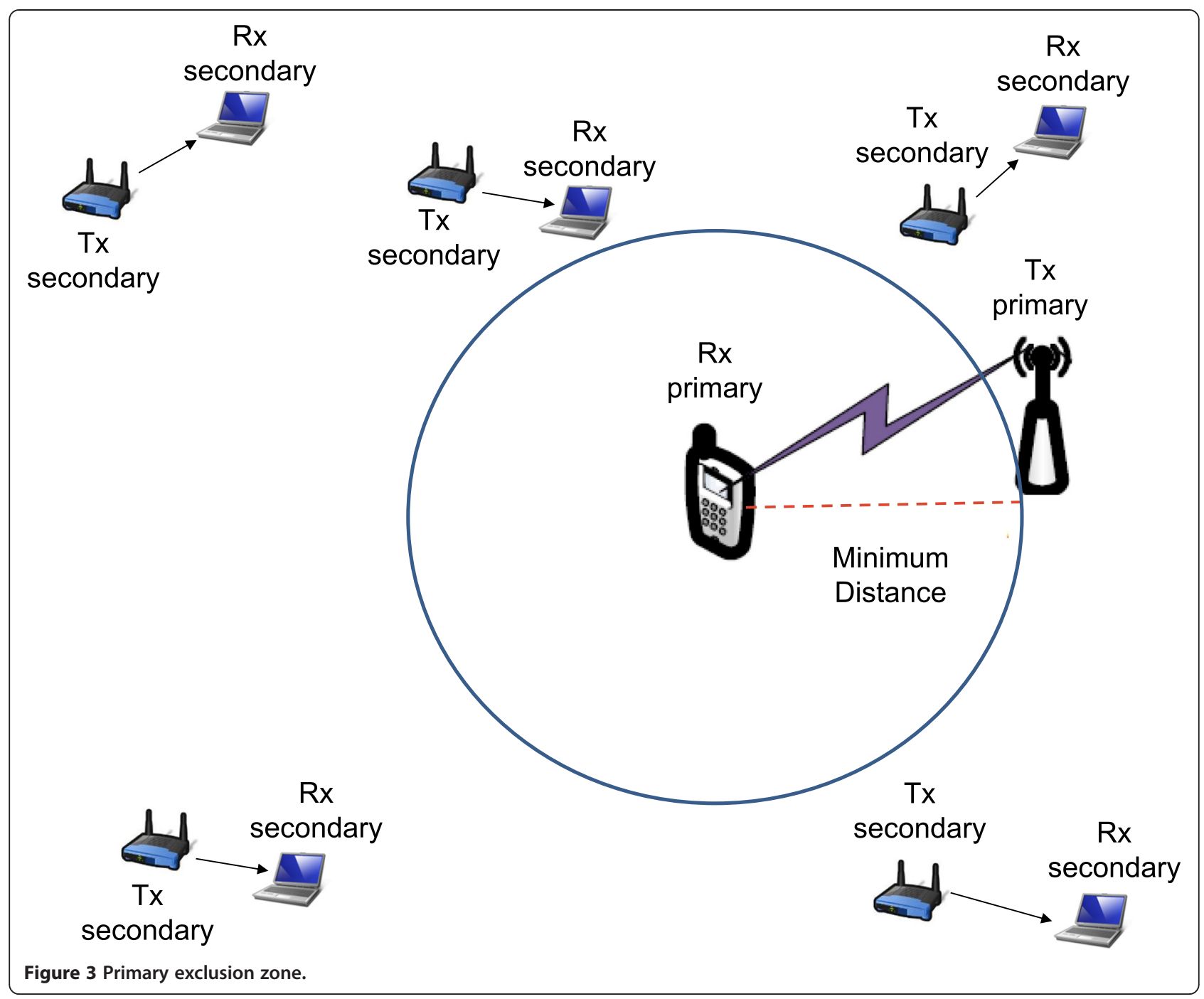

(7) where $\alpha$ and $\beta$ are the SINR thresholds for the secondary network and the primary network, respectively. Constraints from (8) to (10) are integrity restrictions. $x_{u}=1$ if secondary link $u$ is included in the solution and $x_{u}=0$ if it remains out as indicated in (11).

As described in (5) to (11), the goal is to maximize the data rate of the HetNet without degrading the QoS of the primary and secondary networks. To decide which of the deployed secondary links can achieve this, a bio-inspired algorithm called SCPSO is used; that is, from a given scenario, the task of SCPSO is to evaluate different combinations of secondary users deployed in an area along with their primary channels assigned. A combination of secondary users is a candidate solution. In the context of SCPSO, a candidate solution represents a particle $X_{i}$; therefore, the number of particles is called the swarm $S$. Each particle has its own velocity $\left(V_{i}\right)$ to fly through the search space. The particle is able to remember the best position ever visited in the search space since it has a memory $\left(P_{i}\right)$; this position corresponds to the best fitness found by the particle, and it is known as pbest. On the other hand, the best position visited by the whole swarm is called gbest. At each iteration, the particle improves itself according to pbest (its own experience) and gbest (the experience of the best performer in the swarm) until a stop condition is met. Then, gbest is the solution of the problem, that is, the selected secondary users along with their primary channels allocated that achieve the goal.

The $i$ th particle of the swarm $S$ can be represented by a $D$-dimensional vector, $X_{i}=\left(x_{i 1}, x_{i 2}, \ldots, x_{i D}\right), x_{i d} \in\{0,1\}$. The velocity of this particle is represented by another $D$ dimensional vector $V_{i}=\left(v_{i 1}, v_{i 2}, \ldots, v_{i D}\right), v_{i j} \in\left[-V_{\max }, V_{\max }\right]$ where $V_{\max }$ is the maximum velocity. The best previously visited position of the $i$ th particle is denoted as $P_{i}=\left(p_{i 1}\right.$, $\left.p_{i 2}, \ldots, p_{i D}\right), p_{i d} \in\{0,1\}$. Then, the behavior of the whole system emerges from the interaction of particles.

Algorithm 1 describes the access control and channel assignment algorithm using SCPSO as follows: 


\section{Algorithm 1 \\ Initialization stage}

Locate randomly $S l$ and $P l$ over the coverage area $A$

Initialize randomly candidate solution vector $X_{i}$, where $x_{i d} \in\{0,1\}$

Initialize randomly velocity vector $V_{i}$, where $v_{i d} \in\left[-V_{\max }, V_{\max }\right]$

Set $P_{i}=X_{i}$

Initialize randomly candidate channel allocation vector $X_{i}{ }_{i}$ with values from $P l$

Set $P^{\prime}{ }_{i}=X^{\prime}{ }_{i}$

Initialize randomly vector Spectrum Status with values from $P l$

End Initialization stage

1: Loop

2: $\quad$ For $i=1$ to number of particles

3: $\quad$ Perform mapping between $X_{i}{ }_{i}$ and Spectrum Status vectors to calculate SINR levels at secondary and primary links

4: $\quad$ Perform mapping between $P^{\prime}{ }_{i}$ and Spectrum Status vectors to calculate SINR levels at secondary and primary links

5: $\quad$ Calculate the fitness, $f\left(X_{i}\right)$ and $f\left(P_{i}\right)$

6: $\quad$ If $f\left(X_{i}\right)>f\left(P_{i}\right)$ then do

7:

For $d=1$ to $\mathrm{D}$

8:

$$
p_{i d}=x_{i d}
$$

9:

$$
p_{i d}^{\prime}=x_{i d}^{\prime}
$$

10:

Next $d$

11:

End do 


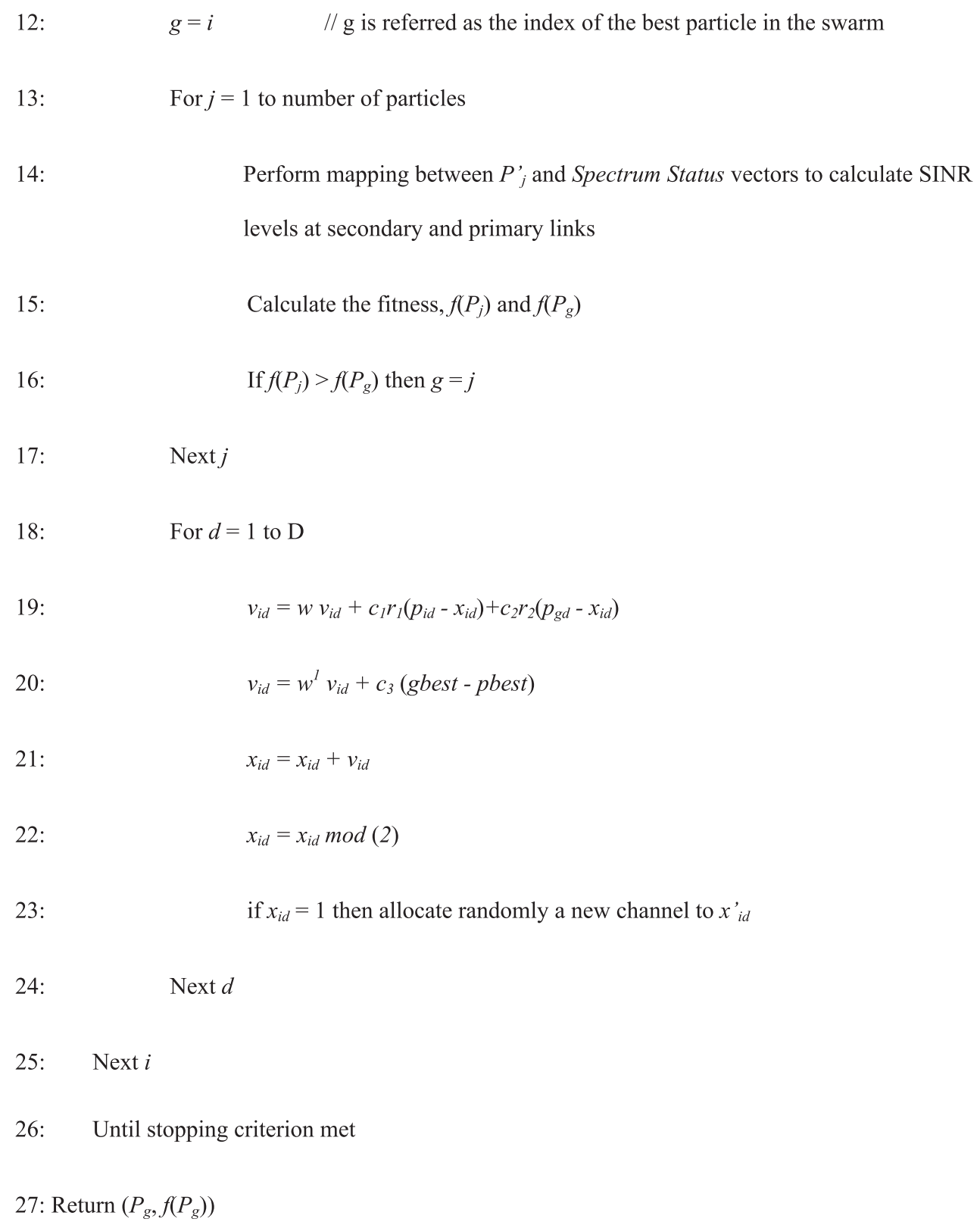

In Algorithm 1, besides the SCPSO vectors $\left(X_{i}, P_{i}\right.$, and $\left.V_{i}\right)$, we include additional vectors related to the $i$ th particle:

- $P_{i}^{\prime}$ which stores the best channel allocation for secondary links find so far.

- $X_{i}$ that represents a candidate channel allocation for secondary links.

- Spectrum status which has the channel allocation for primary links.

Fitness calculation includes the phases of updating pbest and gbest. In update pbest phase (from step 3 to step 11 at Algorithm 1), the particle compares $f\left(X_{i}\right)>f\left(P_{i}\right)$ and overwrites pbest if $f\left(X_{i}\right)$ is higher than $f\left(P_{i}\right)$. In contrast, in update gbest (from step 12 to step 17 at Algorithm 1), all pbest values will be compared with the current gbest value, so if there is a pbest which is higher than the current gbest, then gbest will be overwritten. If the stop condition is not met, $X_{i}$ and $V_{i}$ are updated according to equations from step 19 to step 22 in Algorithm 1; where $c_{1}$ and $c_{2}$ are the learning factors, $c_{3}$ is the socio-cognitive scaling parameter, $r_{1}$ and $r_{2}$ are random numbers uniformly distributed in $[0,1]$, and $w$ and $w^{1}$ are the inertia weights. 
Infeasible solutions, that is, candidate solutions which cannot achieve one or both SINR constraints presented in (6) and (7) are penalized by setting total particle's fitness to zero; therefore, they are not chosen during the process. On the other hand, when conditions (6) and (7) are satisfied, sum data rate is performed as indicated in objective function (5), being the total particle's fitness. Further details and the complete implementation of our solution procedure based on the SCPSO algorithm are provided in [6].

\section{Simulation framework}

Figure 1 shows a snapshot sample (the deployment of secondary links and a primary link in an instant of time) of a particular HetNet that will be analyzed by the access control and channel assignment algorithm using SCPSO to determinate the exclusion zone and the number of selected secondary links which maximize throughput in the HetNet. Due to the distance between different links affects the performance of the HetNet, we considered a stochastic model for the location of the links. The stochastic geometry [13] is a tool for analyzing the distribution of the interference and disruptions that have the links, so that it is possible to statistically analyze network performance. For this, the stochastic models focus on the analysis of a single receiver, or link, as shown in Figure 1, the primary receiver is placed permanently in the center of the coverage area and its corresponding transmitter is located at a distance of $1,000 \mathrm{~m}$. The location of the secondary transmitters is based on the spatial model point Poisson process (PPP) in which each node took a random location represented by a pair of coordinates $\left(a_{i}, b_{i}\right)$.

The metrics for evaluating the performance of the HetNet are directly related to the SINR on links. The SINR depends on many factors such as the distance between the desired transmitter and the desired receiver, and the set of interfering transmitters and their distances from the desired receiver [14]. The SINR is considered in this work by applying Equations (1) and (2) from Section 2, respectively.

During the simulation, several experiments are performed, each of them characterized by a different QoS requirement $(\alpha=\beta)$, then a number of independent runs are taken by each experiment. A run represents a new snapshot with random locations for secondary nodes. Consequently, at each run, different combinations of secondary links coexisting with the primary link are evaluated to find out the best solution, i.e., the combination of secondary links that maximizes throughput in the heterogeneous system. After a number of runs (or snapshots) are taken, the next step is to select the best snapshot; that is, the one with the highest throughput. Then, that snapshot is analyzed to provide the deployment guidelines. Figure 2 summarizes the aforementioned.
Some of the assumptions made for snapshots are:

- The secondary link is located in an area of $25 \mathrm{~km}^{2}$ and its distance is limited to $1,000 \mathrm{~m}$.

- Transmission powers of the primary and secondary are the same.

- The links require the same channel bandwidth since they use the same type of traffic (homogeneous traffic).

- Power attenuation due to multipath and shadowing phenomena is not considered for calculation of SINR.

- For simplicity, the SINR thresholds $\alpha$ and $\beta$ coincide.

The parameter settings for the SCPSO algorithm are shown in Table 2.

The number of experiments depends on $\alpha$ and $\beta$; to analyze, in this work, six experiments are performed, each one representing a different QoS level in the system $(\alpha, \beta=4,6,8,10,12$, and $14 \mathrm{~dB})$. At each experiment, the cases of $10,15,20$, and 25 secondary links along with 1 primary link are evaluated. The configuration parameters for each experiment are shown in Table 3.

The following section shows deployment results from the simulation framework.

\section{Deployment parameters of HetNets}

Through the simulation framework described in the last section, we determine the design parameters that are required for achieving the maximum throughput in the HetNet. The results for the primary exclusion zones and the number of secondary users coexisting with the primary user are shown in the next subsections.

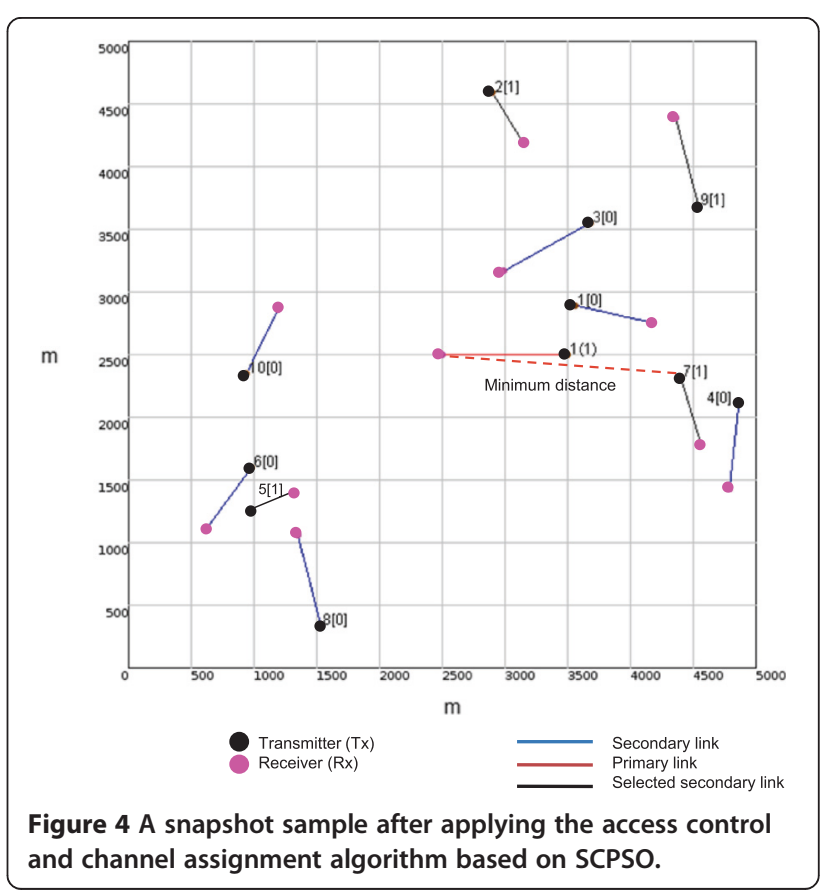




\subsection{Primary exclusion zones}

In the context of HetNets, interference caused by secondary transmitters to the primary receiver should be as low as possible. A solution proposed is to reduce the transmission power of the secondary users. However, this action has an impact on the capacity of the secondary network since the desired signal is affected, decreasing the received SINR. Another solution is to deny transmission to any secondary node when it is in close proximity to a primary receiver. The above is achieved by setting a primary exclusion zone which is the radius of protection for a primary receiver, i.e., the minimum distance to place one or more secondary transmitters with respect to the primary receiver, to use simultaneously the frequency band (see Figure 3).

From Figure 3, it is assumed that each secondary link (transmitter-receiver pair) represents a secondary network or femtocell network. In this case, the secondary transmitter is able to assign a communication channel to its secondary receiver when it requests. Assuming that in an instant of time, the secondary transceiver does not have available channels to assign to its user, it can use its cognitive abilities to exploit any of the primary channels that are assigned to the primary network; so that a primary channel will be allocated to its requested secondary user. Under this situation, the secondary link can exploit concurrently with the primary link the channel, as long as a minimum distance is placed between them; so that the transmission of the secondary link does not interfere with the transmission of the primary link.

After performing the simulation framework described in Section 3, the best snapshot is analyzed by experiment and case to set the primary exclusion zone. Supposing that Figure 4 was the best snapshot, it shows the selected secondary links (black lines) after performing the access control and channel assignment algorithm

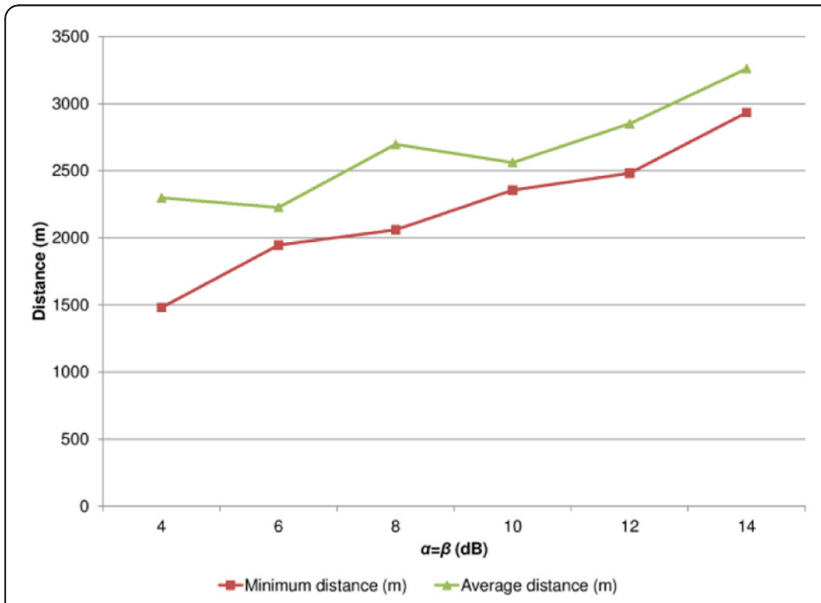

(a)

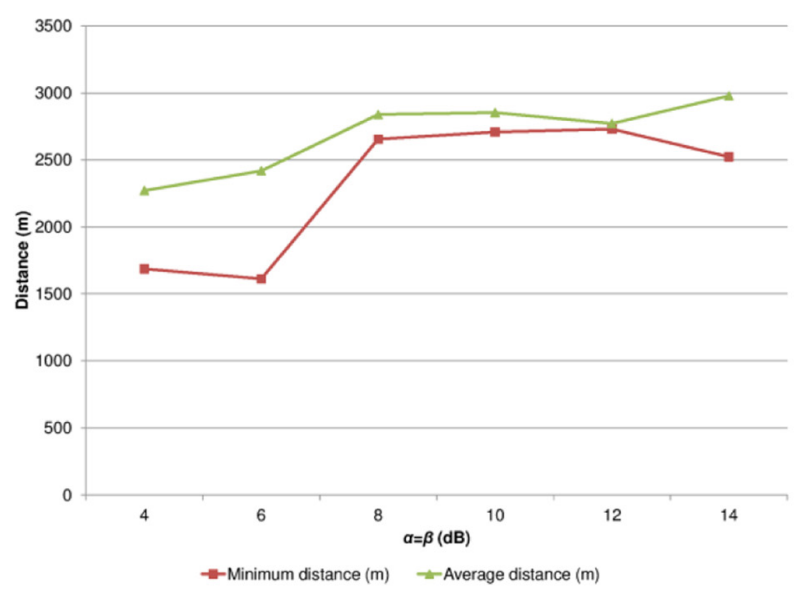

(c)

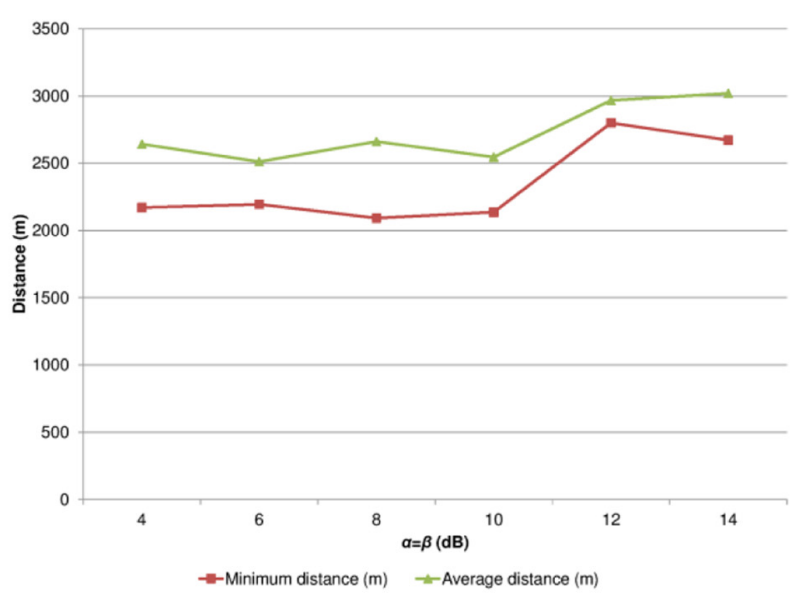

(b)

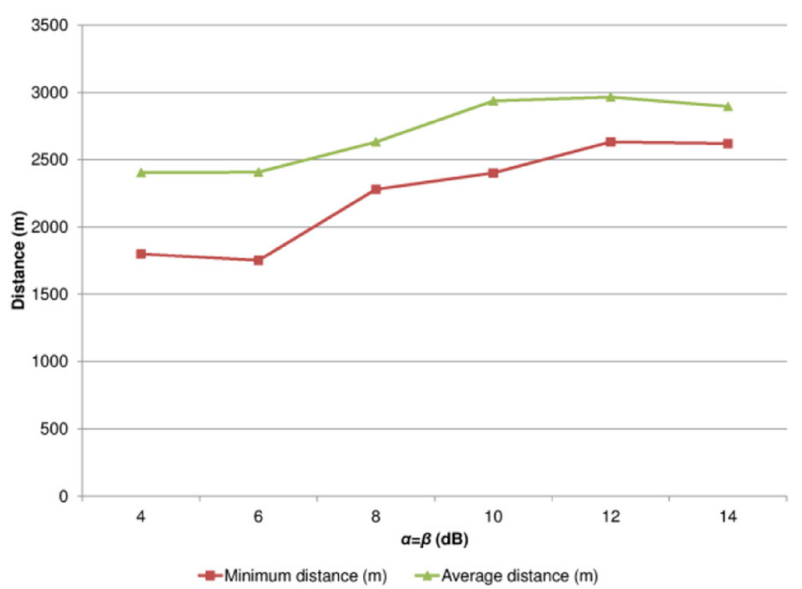

(d)

Figure 5 The minimum and average distances by experiment and case. (a) 10 secondary users, (b) 15 secondary users, (c) 20 secondary users, and (d) 25 secondary users. 
using SCPSO. From the ten secondary users that are requesting for a primary channel, only four of them are selected (secondary links 2, 5, 7, 9). They maximize the throughput of the HetNet. The selected secondary links along with the primary link have the same channel (channel 1). That is indicated in square and rounded brackets for the selected secondary links and the primary link, respectively. Then, the distance between each selected secondary transmitter and the primary receiver is calculated. Among the calculated distances, the shortest distance is selected, and it is considered as the radius of the exclusion zone. Distances are calculated from the location coordinates $\left(a_{i}, b_{i}\right)$ known of each node.

In this context, Figure $5 \mathrm{a}, \mathrm{b}, \mathrm{c}, \mathrm{d}$ shows the values of the minimum (exclusion zone) and average distance that should exist between the secondary transmitters and the primary receiver for different cases (the number of secondary links) and conditions of SINR (the experiments). In this sense, the HetNet performance is not compromised due to mutual interference.

The results in Figure 5a,b,c,d suggest that the higher the QoS level in the HetNet, the longer the distance that should be deployed the secondary transmitters. However, secondary users with lower transmission powers can transmit at a shorter distance from the primary receiver. Those results represent the coverage area (primary exclusion zone) in which a primary receiver can decode the information sent by the primary transmitter without being affected by the interference from secondary links. Outside the coverage area, the secondary links can use the same primary channel to perform their communication, as shown in Figure 3. Then, the radius of the exclusion zone depends on the value of $\alpha$ and $\beta$.

\subsection{Multiple secondary users}

The main challenge in HetNets with multiple secondary users is on how they exploit concurrently a primary channel and take it dynamically. The limiting factor for spectrum reuse is interference; therefore, its control is essential to achieve maximum performance in the HetNet [15]. One of the strategies to achieve the aforementioned is to limit the number of secondary users in the HetNet.

Works [16] and [17] analyze the effect of scaling in wireless networks, that is, quantifying the increase of data rate in the network as the number of links grows. In [16], the authors present results in scaling a homogeneous ad hoc network, determining that the effective throughput of the network is increased by a factor of $\sqrt{n}$, where $n$ represents the number of links in the network. We consider a similar experiment as presented in [17], but unlike that work, we evaluate HetNets which include primary and secondary users. Secondary transmitters are restricted by a distance of $1,000 \mathrm{~m}$ with respect

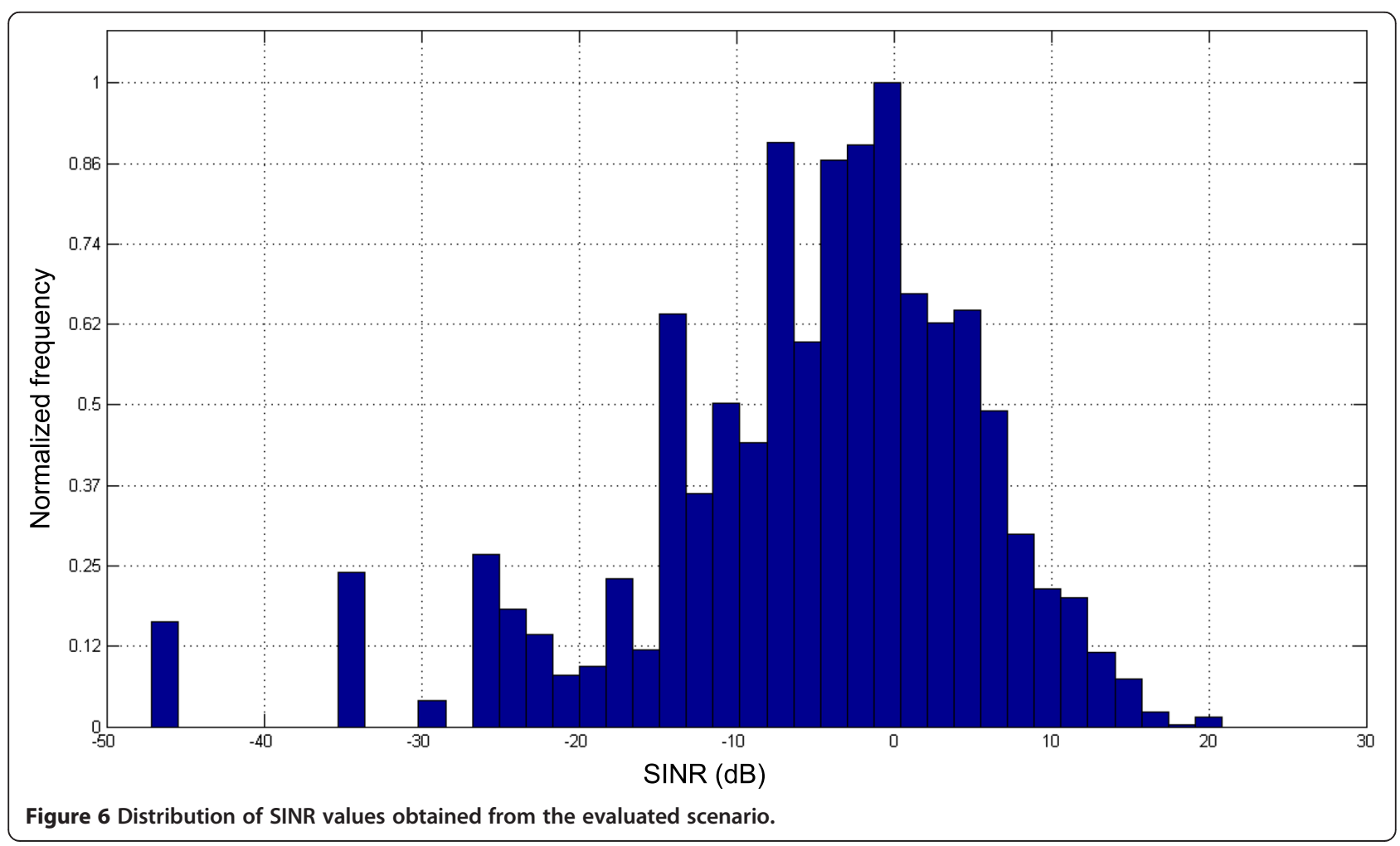


to the secondary receiver. Additionally, our scenario differs from the reported in [16] in which one-hop is considered unlike multiple-hops as in an ad hoc network. Unlike the experiment presented in [17], our work does not evaluate the data rate of the network when the number of secondary links is gradually increased since the access control and channel allocation algorithm is able to select the set of secondary links that meet SINR restrictions.

Figure 6 shows the distribution of SINR values in the primary receiver obtained from our evaluation scenario. It is noted that SINR values are in the range from -46 to $22 \mathrm{~dB}$. This graph shows that the access control and channel allocation algorithm based on SCPSO evaluates different combinations of secondary users to find the best solution.

On the other hand, Figure 7a,b,c,d shows the result of the number of selected secondary links and average number of selected secondary links that can share a channel with the primary user. The number of selected secondary links is derived from the best snapshot by experiment (SINR threshold) and case (number of secondary links); therefore, it corresponds to the minimum distances shown in Figure 5a,b,c,d. In contrast, the average number of selected secondary links is calculated taking into account the number of selected secondary links in each of the 5,000 runs (snapshots) by experiment and case.

From Figure 7a,b,c,d, the observation is as the SINR thresholds values increase, the number of secondary users that the HetNet admits to share a primary channel decreases. On the other hand, when the SINR thresholds values decrease, the HetNet can admit a greater number of secondary users but at the cost of sacrificing QoS. The above is also true, for the average number of selected secondary links, except for the cases of 20 secondary users and 25 secondary users.

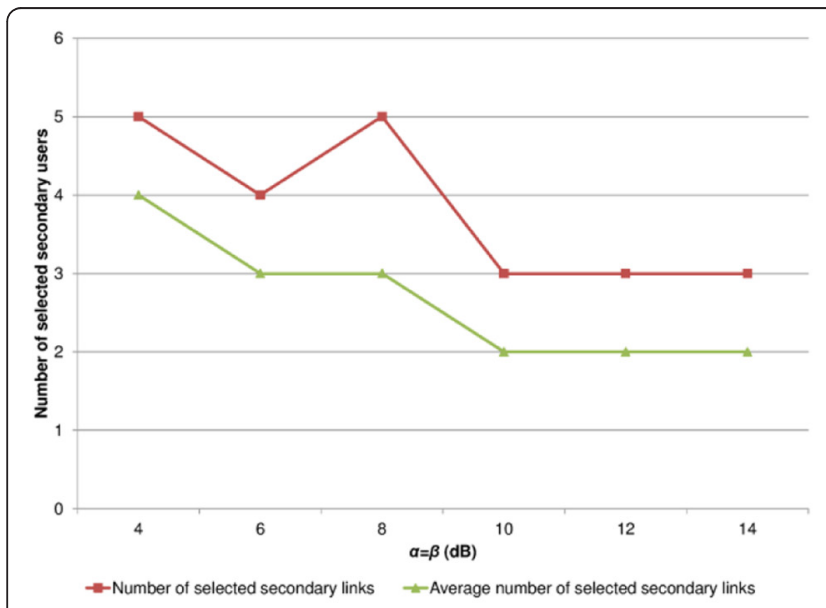

(a)

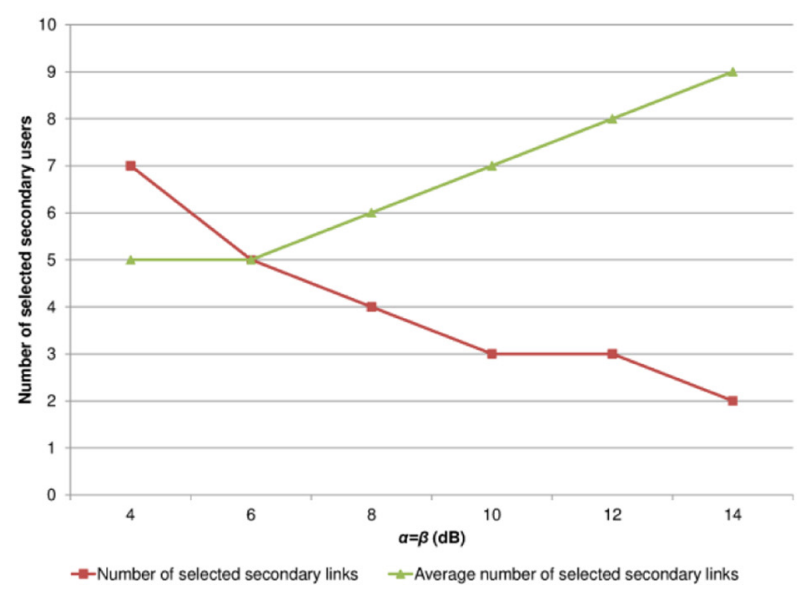

(c)

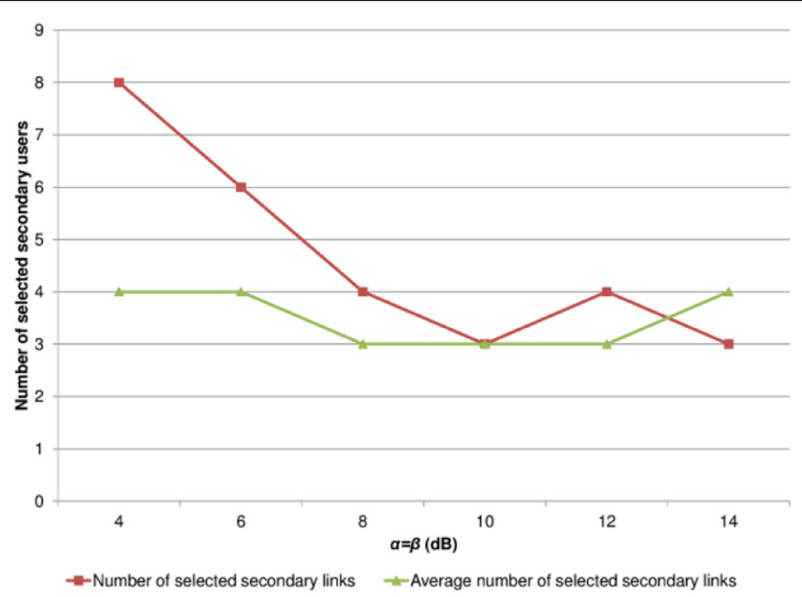

(b)

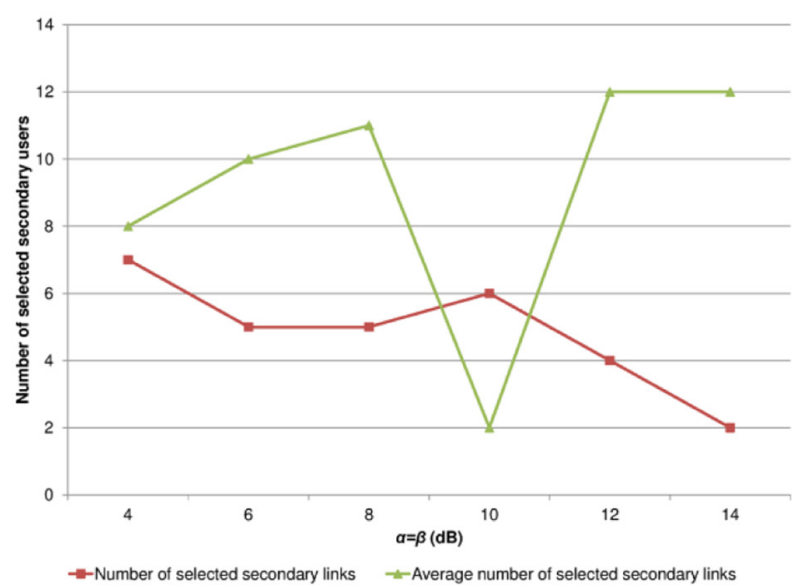

(d)

Figure 7 The number of selected secondary links by experiment and case. (a) 10 secondary users, (b) 15 secondary users, (c) 20 secondary users, and (d) 25 secondary users. 
The benefit of the HetNet is the reuse of spectrum. However, the risks are equally important to consider, for example to control the number of admitted secondary users to coexist with the primary user. If the above is not controlled, it can lead to a 'tragedy of the commons' in which many users try to access the same spectral resource and neither of them is able to communicate due to the interference. A higher permissible density of secondary users also represents a more efficient reuse [18]. These facts show the importance for providers and regulators in the tradeoff between the permissible density of secondary users and the SINR threshold (QoS).

We will now present system performance results in terms of data rate. Figure 8a,b,c,d shows the maximum and average data rate that the system achieves by experiment and case. The maximum data rate of the system is the best found, i.e., the run (or snapshot) with the highest data rate from the set of 5,000 runs. Those maximum data rates corresponds to the minimum distance and the number of selected secondary users from Figures 5a,b,c,d and 7a,b,c, $\mathrm{d}$, respectively. In contrast, the average maximum data rate is obtained from the maximum data rates of each of the 5,000 runs. Results from Figure 8a,b,c,d suggest that the higher the SINR threshold values, the lower the data rate. This observation is consistent with results reported in [19]: the throughput is degraded significantly when the interference constraint is made more stringent; therefore, the performance of the underlay approach depends mainly on the interference constraint.

From those challenging scenarios of 20 and 25 secondary users (Figure $8 \mathrm{c}, \mathrm{d}$ ), the average maximum data rate tends to decrease dramatically, especially when the QoS requirements are higher. That implies that as the number of users increases, the interference also arises; making more challenging for the SCPSO to converge or to find a solution. To address that issue, a future work is to propose different

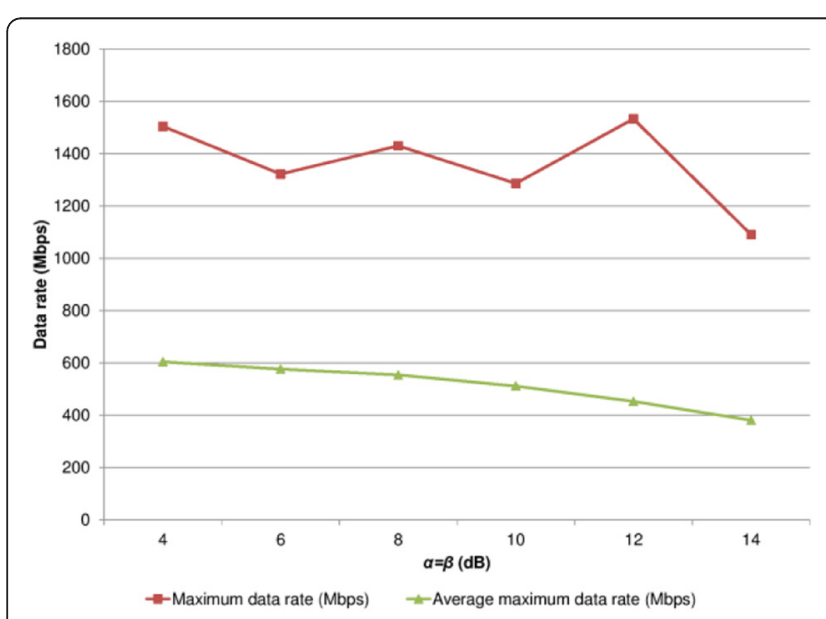

(a)

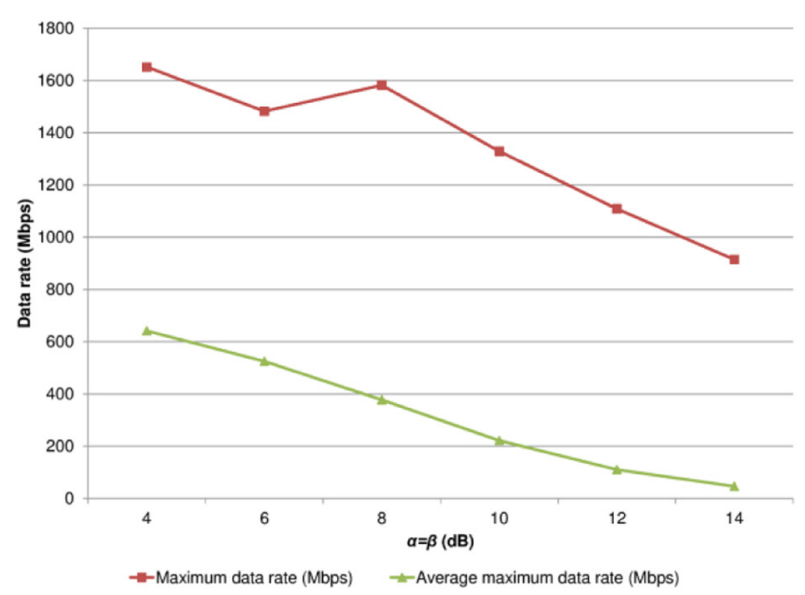

(c)

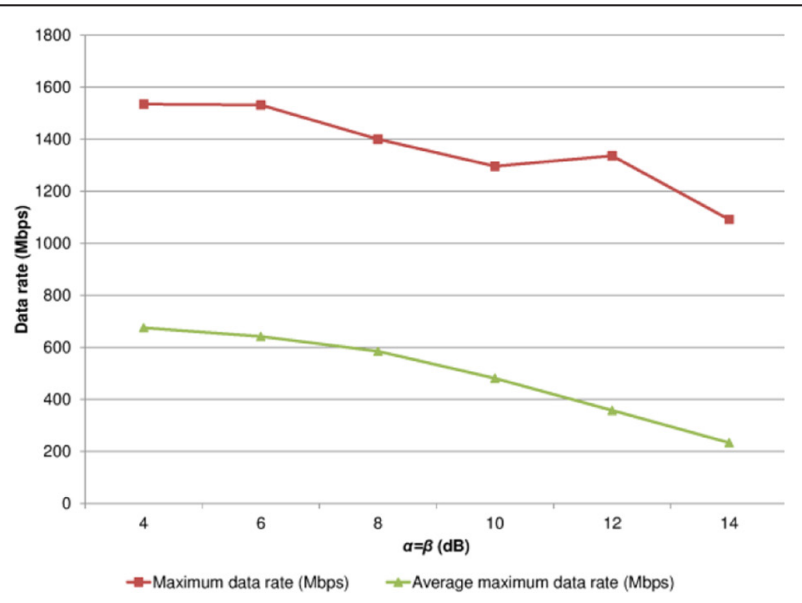

(b)

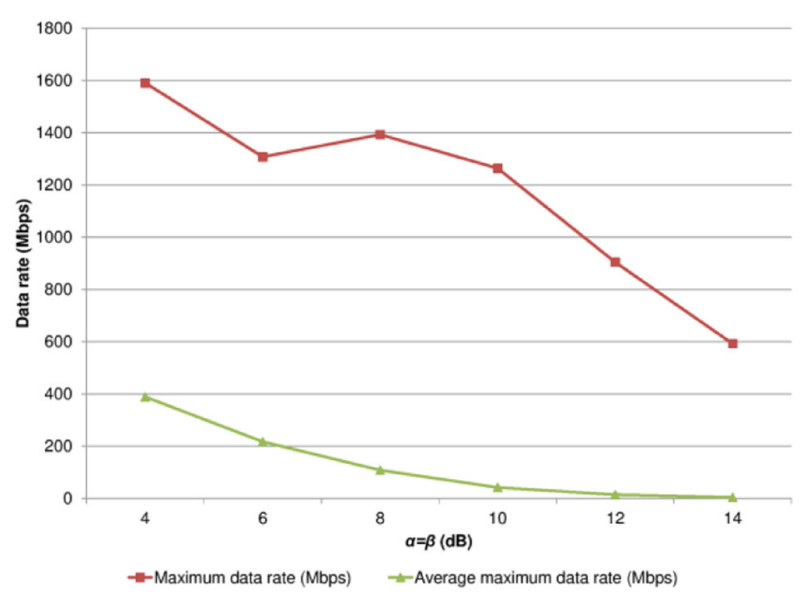

(d)

Figure 8 The maximum and average data rate by experiment and case. (a) 10 secondary users, (b) 15 secondary users, (c) 20 secondary users, and (d) 25 secondary users. 


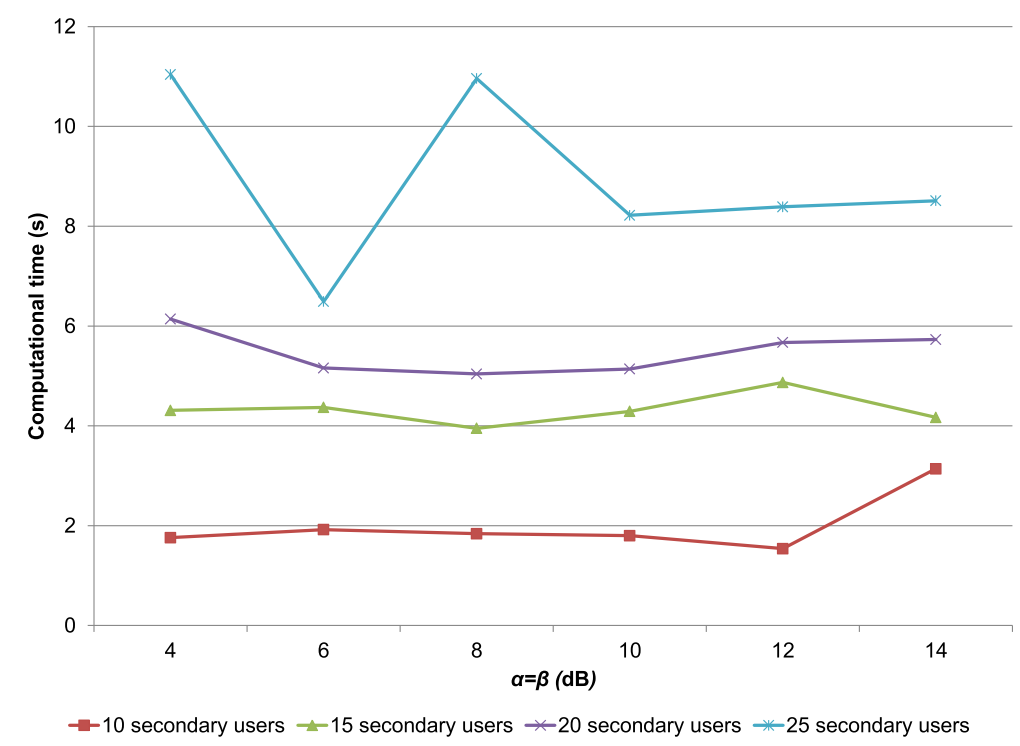

Figure 9 Computational time for the maximum data rates found.

parameters for the SCPSO by experiment and case, since keeping them fixed for all the experiments (the SINR thresholds) and cases (the number of secondary links) is not the best strategy to optimize its performance in challenging scenarios.

On the other hand, Figure 9 gives further insight into the computational time from those maximum data rates reported in Figure 8a,b,c,d. The running time is in various seconds, even in the most challenging scenarios such as in the cases of 20 and 25 secondary users. It increases with the number of secondary users deployed in the area and the SINR thresholds.
Finally, Figures 10 and 11 depict the convergence of average data rate through iterations, that is, considering the maximum data rates of each of the 5,000 runs. In Figure 10, the experiment of $4 \mathrm{~dB}$ for the cases of 10 and 25 secondary users is considered, which represents the less challenging scenario. In contrast, in Figure 11, the experiment of $14 \mathrm{~dB}$ for the cases of 10 and 25 secondary users, that is the most challenging scenario, is considered. From Figure 10, we observe that the access control and channel assignment algorithm using SCPSO takes more iteration to converge when there are more secondary users deployed in the area. That insight is

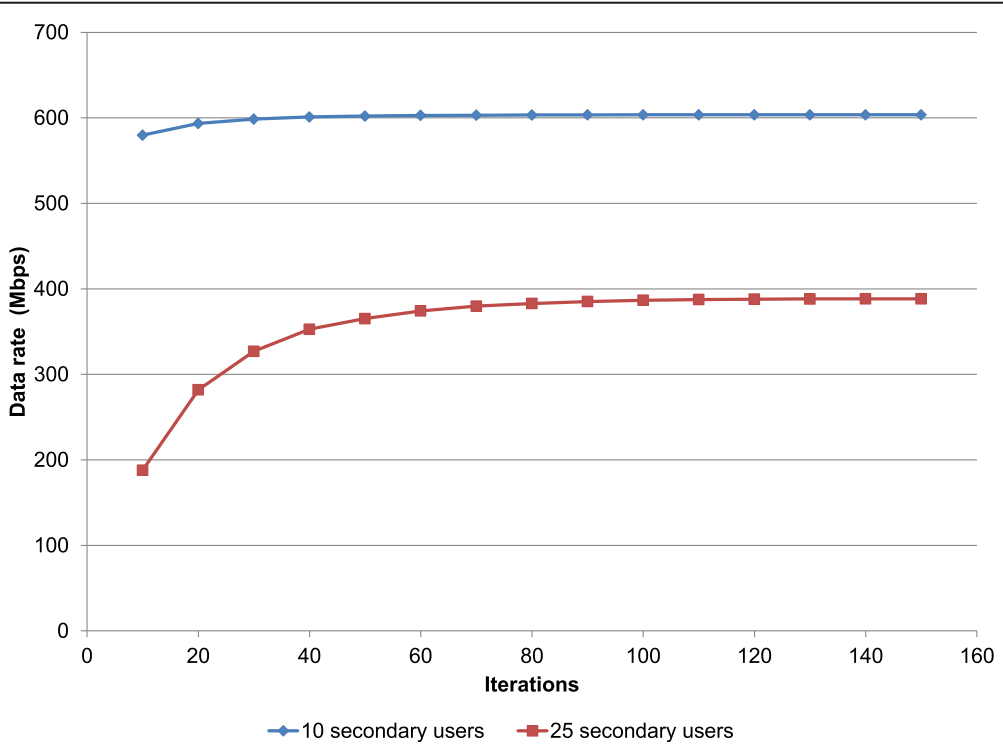

Figure 10 Convergence of average data rate (experiment $=4 \mathrm{~dB}$, cases $=10,25$ secondary users). 


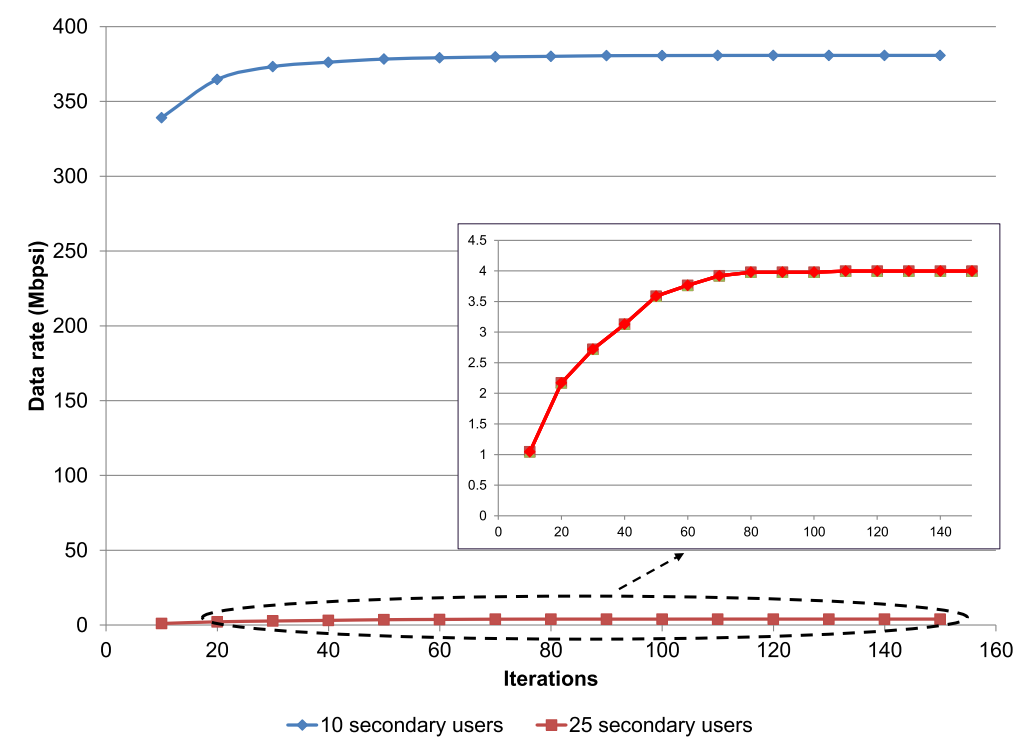

Figure 11 Convergence of average data rate (experiment $=14 \mathrm{~dB}$, cases $=10,25$ secondary users).

also true for challenging scenarios such as that described in Figure 11; the average data rate is low in the case of 25 users. The above is consistent with the observation identified in Figure 8a,b,c,d, about to set different parameters for SCPSO by experiment and case to optimize its performance to find a solution.

\section{Conclusions}

In this work, we presented a framework to calculate the radius of the exclusion zone and the permissible density of secondary users to achieve the maximum throughput in the HetNet.

The interference from the secondary users to the primary user can be limited by defining a protection distance (primary exclusion zone). In this context, from simulation was observed that the higher the QoS level in the HetNet, the longer the distance that should be deployed in the secondary transmitters.

Simulation results also showed that from the number of secondary users wishing to operate concurrently with the primary user, a certain number of secondary users will be accepted depending on the QoS requirement.

Even when the computational time for a run was in seconds, a main issue was the parameter selection (tuning) on SCPSO algorithm. Future work will focus on identifying parameter values that improve the search ability of SCPSO to find a solution in challenging scenarios, since keeping them fixed for all the experiments and cases was not the best strategy to optimize its performance on the HetNet application.

\section{Competing interests}

The authors declare that they have no competing interests.
Received: 1 July 2014 Accepted: 9 February 2015

Published online: 10 March 2015

\section{References}

1. N Himayat, S Yeh, AY Panah, S Talwar, M Gerasimenko, S Andreev, Y Koucheryavy, Proceedings of the IEEE International Conference on Computing, Networking and Communications (ICNC). Multi-radio heterogeneous networks: Architectures and performance (IEEE Honolulu, USA, 2014), pp. 252-258

2. SM Cheng, WC Ao, FM Tseng, KC Chen, Design and analysis of downlink spectrum sharing in two-tier cognitive femto networks. IEEE Trans. Veh. Technol. 61(5), 2194-2207 (2012). doi:10.1109/TVT.2012.2187692

3. Y Liu, LX Cai, X Shen, H Luo, Deploying cognitive cellular networks under dynamic resource management. IEEE Wirel. Commun 20, 82-88 (2013). doi:10.1109/MWC.2013.6507398

4. C Jiang, $H$ Jiang, C Jiang, S Fan, Y Ren, C Chen, Proceedings of the IEEE Military Communications Conference (MILCOM). Analysis on deployment parameters of secondary users in cognitive radio networks (IEEE San Jose, USA, 2010), pp. 743-748

5. MF Hanif, M Shafi, PJ Smith, P Dmochowski, Proceedings of the IEEE International Conference on Communications (ICC). Interference and Deployment Issues for Cognitive Radio Systems in Shadowing Environments (IEEE Dresden, Germany, 2009), pp. 1-6

6. A Martínez-Vargas, ÁG Andrade, R Sepúlveda, O Montiel-Ross, in Recent Advances on Hybrid Approaches for Designing Intelligent Systems, ed. by O Castillo, P Melin, W Pedrycz, J. Kacprzyk, (Springer International Publishing Switzerland, 2014), p. 151

7. K Deep, JC Bansal, Proceedings of the IEEE International Conference on Emerging Trends in Engineering and Technology (ICETET). A Socio-Cognitive Particle Swarm Optimization for Multi-Dimensional Knapsack Problem (IEEE Nagpur, India, 2008), pp. 355-360

8. MF Hanif, PJ Smith, PA Dmochowski, Statistical interference modelling and deployment issues for cognitive radio systems in shadow fading environments. IET Commun. 6, 1920-1929 (2012)

9. O Durowoju, K Arshad, K Moessner, Proceedings of the IEEE Vehicular Technology Conference (VTC Fall). Distributed Power Control and User Selection Algorithms for Cognitive Radios (IEEE Las Vegas, USA, 2013), pp. 1-5

10. R Dahama, KW Sowerby, GB Rowe, Estimating protection distances in spectrum sharing systems. IEEE Trans. Signal Process. 61, 4284-4295 (2013)

11. P Tian, H Tian, L Gao, J Wang, X She, L Chen, Proceedings of the IEEE International Symposium on Wireless Personal Multimedia Communications (WPMC). Deployment analysis and optimization of Macro-Pico heterogeneous networks in LTE-A system (IEEE Taipei, Taiwan, 2012), pp. 246-250 
12. C Avin, Y Emek, E Kantor, Z Lotker, D Peleg, L Roditty, SINR diagrams: convexity and its applications in wireless networks. JACM 59(18), 1-18 (2012). 34. doi:10.1145/2339123.2339125

13. RK Ganti, M Haenggi, Interference and outage in clustered wireless ad hoc networks. IEEE Trans Inf Theor 55, 4067-4086 (2009)

14. JG Andrews, RK Ganti, M Haenggi, N Jindal, S Weber, A primer on spatial modeling and analysis in wireless networks. IEEE Commun. Mag. 48, 156-163 (2010)

15. EZ Tragos, S Zeadally, AG Fragkiadakis, VA Siris, Spectrum assignment in cognitive radio networks: a comprehensive survey. IEEE Commun Surv Tutor 15, 1108-1135 (2013)

16. P Gupta, PR Kumar, Towards an information theory of large networks: an achievable rate region. IEEE Trans. Inf. Theory 49, 1877-1894 (2003)

17. $M V$ Vu, N Devroye, M Sharif, $V$ Tarokh, in Proceedings of the IEEE International Conference on Cognitive Radio Oriented Wireless Networks and Communications (CrownCom) (USA, Scaling Laws of Cognitive Networks (IEEE Orlando, 2007). pp. 2-8

18. J Lee, JG Andrews, D Hong, Spectrum-sharing transmission capacity. IEEE Trans. Wirel. Commun. 10, 3053-3063 (2011)

19. A Alsarhan, A Agarwal, I Obeidat, M Bsoul, A Khasawneh, Y Kilani, Optimal spectrum utilization in cognitive network using combined spectrum sharing approach: overlay, underlay and trading. Int J Bus Inform Syst 12, 423-454 (2013)

\section{Submit your manuscript to a SpringerOpen ${ }^{\circ}$} journal and benefit from:

- Convenient online submission

- Rigorous peer review

- Immediate publication on acceptance

- Open access: articles freely available online

- High visibility within the field

- Retaining the copyright to your article 